\title{
Be careful eating the local catch
}

\section{An unusual case of mass scombroid poisoning in deployed forces in Indonesia}

\author{
Eric Roszell (Meds 2016), Craig Olmstead (Meds 2017) \\ Faculty Reviewer: Dr William Moote, MD, FRCPC (Department of Medicine, Division of Clinical Immunology \& Allergy)
}

\section{INTRODUCTION}

Our case begins in a setting far removed from a typical North American hospital. It begins in Sumatra, Indonesia during earthquake recovery efforts in November, 2009. An Australian Defence Force medical team was deployed to help with the recovery efforts. After a successful mission providing relief, the deployed forces started to pack up their supplies and prepared to leave in the next few days. Shortly after dinnertime, however, one of the staff members presented to the medical staff in the mobile centre with generalized urticaria. $^{1}$

\section{INITIAL CONSIDERATIONS \& RESPONSE}

Acute urticaria, a red itchy rash often referred to as hives, can have multiple etiologies. The most common causes are related to allergic reactions to foods, medications, or insect bites. Although more common in pediatric patients, infections can also lead to acute urticaria in adults. Rarer causes for adult urticarial include vasculitis and systemic lupus erythematosus, but these usually present with other physical symptoms. ${ }^{2}$

Due to the limited resources of the mobile centre and acute time frame of presentation, full testing could not be completed, so these rarer causes could not be completely ruled out. Based on the presentation of acute urticaria in isolation, a tentative diagnosis of an allergic reaction was given for the staff member and a search was done to elucidate the trigger for the reaction. Intramuscular adrenaline, oral promethazine, ranitidine, and intravenous steroids were administered. Before a trigger for the allergic reaction could be determined, however, another patient presented with a similar clinical picture.

\section{ADDITIONAL CASES}

A second staff member presented with truncal urticaria, oral angioedema, hoarseness, and dysphagia approximately $20 \mathrm{~min}$ utes after the first patient had sought assistance. ${ }^{1}$ The addition of a second case to this clinical picture so soon after the initial case, while not inconsistent with an allergic reaction, raised doubts about whether a hypersensitivity reaction was truly the underlying cause. ${ }^{3}$ This second staff member mentioned that some others were also feeling unwell and experiencing similar symptoms. Over another 20 minutes, 5 other staff members presented with varying degrees of urticaria, erythema, abdominal pain, and dysphagia. Given the setting-a mobile Australian Defence Force base in Indonesiathis massive influx of new patients had several important implications. Due to the small size of the mobile centre and because many of the supplies had been packed, the treatment capacity of the centre was quickly overwhelmed by this sudden influx of patients. The supply of adrenaline and steroids was quickly exhausted, and there were only two patient monitors available. To further compound this resource shortage, one of the senior nurses also began to show symptoms. Panic set in among the staff as they wondered about the identity of the trigger for these reactions. The search for a causative agent was intensified. The patients had some similar exposures that were investigated, such as a wet muddy field and a lightly damaged building they had visited previously; however, no causative agent could be found. Ingested toxins were also considered but none were identified at this point. ${ }^{1}$

\section{RESPONSE \& RESOLUTION}

As no causative agent could be found, evacuation of the patients to a nearby offshore ship, the HMAS (Her Majesty's Australian Ship) Kanimbla, was requested. Medical staff on board the ship were informed of the situation and proceeded to conduct an internet search for possible causative agents. That search suggested the possibility of scombroid poisoning, a type of food poisoning associated with the consumption of fish. Further investigation revealed all affected patients had eaten 3 servings or more of a locally acquired white meat dish believed to contain fish. This dish was referred to as "ficken" by the Australian Defence Force medical team as a joke because of the inability to ascertain exactly what kind of meat it was made from. Upon questioning, other staff personnel who ingested smaller quantities of ficken reported some mild nausea and abdominal cramping. Based on these findings, a clinical diagnosis of scombroid toxicity was made and all patients were successfully treated with intramuscular adrenaline, ranitidine, intravenous steroids, and oral promethazine. Additional supplies and personnel were dispatched from the HMAS Kanimbla to assist with treatment. ${ }^{1}$

\section{SCOMBROID POISONING}

Scombroid food poisoning generally occurs when histidine-rich fish are consumed after improper storage. ${ }^{1}$ Fish of the Scombroidei genus, such as tuna or mackerel, are the most common culprits, though other species fish have been implicated.1,4,5 The pathogenesis of scombroid poisoning is believed to be the conversion of histidine to histamine by bacteria, often gram-negative enteric bacilli such as Escherichia coli. ${ }^{1,6}$ The conversion process involves a decarboxylase enzyme, and occurs primarily when the fish is stored above $16^{\circ} \mathrm{C} .{ }^{1}$ Other biogenic amines may play a role, though their effects have yet to be established. ${ }^{1,5}$ Nevertheless, the result is, in effect, poisoning by an overabundance of histamine. ${ }^{1,3-5}$ Symptoms vary, but are extremely similar to an immediate IgE-mediated hypersensitivity reaction and can include flushing, widespread urticaria, edema, nausea, and bronchospasm. ${ }^{1,3,7}$ Initial onset of symptoms often occurs within one hour of consumption of the affected food, and can persist for up to 36 
hours. ${ }^{1,4}$ Scombroid food poisoning can be extremely difficult to diagnose, due to the similarity of its symptoms to those of an allergic reaction and its atypical method of action. While scombroid poisoning does involve bacterial spoiling of food, unlike more common forms of food poisoning, killing the responsible bacteria does not eliminate the toxin-high heat does not destroy histamine, so properly cooked fish can still cause scombroid poisoning. ${ }^{1,5}$ Furthermore, there may be no obvious signs of spoiling, as histamine does not change the odor or appearance of the fish. ${ }^{4}$

Diagnosis of scombroid poisoning can often be made clinically, though additional testing may be used for confirmation. ${ }^{1,6,7}$ The timing of symptoms, with an initial presentation shortly after consumption, is an important factor but may not help distinguish scombroid from a standard allergic reaction. ${ }^{5}$ The keys to clinical diagnosis are the absence of a history with allergies and the simultaneous onset of symptoms with other patients. ${ }^{1,5-7}$ Blood histamine levels will be highly elevated initially, with a significant reduction within 24 hours. ${ }^{6}$ Levels of histamine or histamine byproducts in the urine may also be elevated. ${ }^{6}$ If a sample is available, direct testing of the ingested fish for histamine concentrations can also be diagnostic. ${ }^{3,6}$ In our case, the situation precluded use of supplementary diagnostic tools, and the diagnosis was made clinically. ${ }^{1}$

Treatment for scombroid poisoning is supportive in nature. ${ }^{1,7}$ Fast-acting antihistamines, fluids, and antipyretic agents should be used to control symptoms as needed. ${ }^{1,4,7}$ Promethazine, the antihistamine used in this case, is an older, first generation sedating antihistamine that is not frequently used in Canada; diphenhydramine is traditionally the emergency antihistamine of choice. In extreme cases, where closure of the airway becomes a concern, administration of adrenaline may be necessary. ${ }^{1,7}$ Use of corticosteroids has been reported and was used in this case, although their value has been questioned. ${ }^{1,3,47}$ Patients should recover within 36 hours as the excess histamine is cleared from the body, though careful monitoring is required to ensure symptoms are responding appropriately to medication., ${ }^{1,3-5}$

\section{CONCLUSION}

This case illustrates some of the challenges of practicing medicine in a more remote area with limited supplies and resources, and where backup cannot be accessed quickly. Treatment of patients was initially hindered both because of a supply shortage and because health care providers who would normally provide treatment were among the patients. Assistance eventually was provided by the HMAS Kanimbla, but there was a 4-hour delay between the request for assistance and help arriving. The lack of medical and human resources made the search for the causative agent more difficult as well. This is highlighted by the fact that foodborne illness was considered but initially ruled out due to inadequate investigation. Moreover, ignorance about the regional cuisine-in this case, the mystery meat in ficken-proved to be a barrier in establishing a diagnosis, as the common history of fish consumption was revealed only after scombroid poisoning was proposed as a possibility, despite a concerted effort to find a connection between patients. This highlights how practicing medicine in unfamiliar locations or cultures can result in missing knowledge, and therefore lead to an incomplete history.
However, this case also demonstrates the value of open lines of communication. The ability to consult outside support and subsequently to call in additional resources was instrumental in achieving a successful response to this unique medical crisis. ${ }^{1}$ The insights gained from this case are relevant to medicine practiced in situations with limited resources and in situations where information is limited due to a culture gap. These lessons are increasingly important as medicine continues to be practiced from a more global perspective.

\section{REFERENCES}

1. Ward DI. 'Mass allergy': acute scombroid poisoning in a deployed Australian Defence Force health facility. Emerg Med Australas. 2011 Feb;23(1):98-102.

2. Peroni A, Colato C, Schena D, Girolomoni G. Urticarial lesions: if not urticaria, what else? The differential diagnosis of urticaria: part I. Cutaneous Diseases. J Am Acad Dermatol. 2010 Apr;62(4):541-55.

3. Jantschitsch C, Kinaciyan T, Manafi M, Safer M, Tanew A. Severe scombroid fish poisoning: an underrecognized dermatologic emergency [Letter]. J Am Acad Dermatol. 2011 Jul;65(1):246-7.

4. Stratta P, Badino G. Five things to know about scombroid poisoning. Can Med Assoc J. 2012 Apr;184(6):674.

5. Hungerford JM. Scombroid poisoning: a review. Toxicon. 2010 Feb;56:231-43.

6. Bédry R, Gabinski C, Paty M. Diagnosis of scombroid poisoning by measurement of plasma histamine [Letter]. N Engl J Med. 2000 Feb; 342(7):520-1.

7. Vickers J, Safai B. Images in clinical medicine: scombroid poisoning. N Engl J Med. 2013 Jun;368(23):e31. 